\title{
Tuberculous mycobacteria bacilli fluorescence and compare with Ziehl- Neelsen stain in fine-needle aspiration cytology of tubercular lymphnode
}

\author{
Mani Krishna ${ }^{1}$, Adesh Kumar ${ }^{2}$ \\ ${ }^{1}$ Department of Pathology, ${ }^{2}$ Department of Pulmonary Medicine, U.P. RIMS \& R Saifai Etawah, Uttar Pradesh, India
}

Received: 30 December 2015

Accepted: 04 February 2016

*Correspondence:

Dr. Mani Krishna,

E-mail: drmanikrishna@gmail.com

Copyright: $\odot$ the author(s), publisher and licensee Medip Academy. This is an open-access article distributed under the terms of the Creative Commons Attribution Non-Commercial License, which permits unrestricted non-commercial use, distribution, and reproduction in any medium, provided the original work is properly cited.

\begin{abstract}
Background: Tuberculosis is infectious disease caused by Mycobacterium tuberculosis. There are various methods for diagnosis of tuberculosis such as direct clinical material examination of tubercular bacilli by Ziehl - Neelsen (ZN) staining, demonstration of tubercular bacilli by auramine - rhodamine (AR) staining and autofluorescence (AF).

Methods: Present study was done clinically suspected tubercular patients. All received samples Zn stain, fluorescent stain and PAP stain were applied.

Results: Among the clinically suspected patients 88 was diagnosed with tuberculosis. Female preponderance was noted accounting for $60.23 \%$ (53/88) of cases. Of the 88 aspirates, the smear positivity for acid fast bacilli (AFB) on the $\mathrm{ZN}$ method was $37.5 \%$ (33/88) while the positivity increased to $81.82 \%(72 / 88)$ on the AR fluorescent method and $86.36 \%(76 / 88)$ on AF.

Conclusions: AF staining is more sensitive than the auramine - rhodamine fluorescent and $\mathrm{ZN}$ staining in demonstration of mycobacterium bacilli in fine needle aspiration cytology of tubercular lymphnode.
\end{abstract}

Keywords: Tuberculosis, Mycobacterium tuberculosis, AFB, ZN staining, AR staining, AF

\section{INTRODUCTION}

Tuberculosis continues to be a major health problem in developing countries. Tuberculosis is one of the oldest diseases known to affect humans and is caused by Mycobacterium tuberculosis. Lymphadenopathy is the most common presentation of extra-pulmonary tuberculosis. $^{1,2}$ Tuberculous lymphadenitis can be presumptively diagnosed morphologically on fine-needle aspiration cytology of lymph node. Fine-needle aspiration cytology is now widely utilized as a first line diagnostic procedure in the diagnosis of palpable masses, including peripheral lymphadenopathy. Its value in the diagnosis of mycobacterial lymphadenitis in adults is well documented. Fine-needle aspiration cytology is a simple effective and safe modality for obtaining a representative sample of material from a lymph node and the diagnosis of mycobacterial adenitis can be confirmed utilising a number of different investigations, including cytomorphology, specific stains to identify the organism, culture and polymerase chain reaction. However, culture is essential for obtaining a definitive diagnosis. Unfortunately, culture is time consuming and expensive. Koch first described the tubercle bacilli in 1882 which is now known to Mycobacterium tuberculosis. Mycobacteria are now known to comprise a large group of acid - fast, alcohol - fast, aerobic or microaerophilic, non - spore forming, non-motile bacilli. ${ }^{3}$ However, the Ziel-Neelsen method for acid-fast bacilli plays a key role in the diagnosis and also for the monitoring of treatment in tuberculosis. Its major disadvantage is low sensitivity 
ranging from $20 \%$ to $43 \% .^{4,5}$ Serological techniques have the disadvantage of lack of sensitivity and specificity. ${ }^{4}$ Newer molecular techniques such as polymerase chain reaction, although rapid, are costly to be routinely used in developing countries where most TB cases occur. ${ }^{6}$ Newer investigative methods are required. Fluorescent microscopy plays an important role for detection of Mycobacteria because lower magnifications are used as well as less time is required to examine smears. Flurescence microscopy using auramine-rhodamine (AR) or papanicolaou (PAP) staining has been considered to be superior to $\mathrm{ZN}$ staining. ${ }^{7,8}$ The method is quick and inexpensive. The efficacy of autofluorescence and fluorescence in the diagnosis of tuberculous lymphadenitis was evaluated for this purpose.

\section{METHODS}

This study was conducted in department of Rural Institute of Medical Sciences and research Saifai Etawah (U.P), India from January to July 2015. A total of 212 patients having peripheral lymphadenopathy were referred for FNAC to cytology lab of the department of pathology. Four smears were made from each aspirate: three air dried smears were stained with Giemsa, ZN, and AR stains and one was wet fixed for PAP stain for autofluorescence. AR and PAP stained slides were examined under fluorescent microscope. By $\mathrm{ZN}$ stain AFB stain pink curve or straight beaded rods against blue background and by AR stain AFB appears as bright reddish-yellow fluorescing rods against a dark background and by PAP stain AFB stained mycobacteria fluoresce as brilliant yellow bacilli, thin and slightly curved.

\section{RESULTS}

A total of 212 fine-needle aspirated specimens from lymph nodes were included in the study. Out of 212 cases, 88 aspirates were reported as cytomorphology suggestive of tuberculous lymphadenitis. The age ranged from 1 to 70 years, with the mean age of 35.5 years. Female preponderance was noted accounting for $60.23 \%$ (53/88) of cases (Table 1). 68.18\% (60/88) of the cases with suggestive cytomorphology of tubercular lymphadenitis were in the range of 11-30 years of age. Depending upon cytomorphological features, granuloma $75(85.23 \%)$, necrosis $70(79.55 \%)$, acute inflammation $25(28.41 \%)$, lymphoid background 21 (23.86\%), giant cell $15(17.05 \%)$ were found in tuberculous lymphadenitis (Table 3). In present study, the most common site of involved lymph nodes was of the cervical region in $64.77 \%(57 / 88)$ of the cases (Table-2). Of the 88 aspirates, the smear positivity for AFB on the $\mathrm{ZN}$ method was $37.5 \%(33 / 88)$ while the positivity increased to $81.82 \%(72 / 88)$ on the AR fluorescent method and $86.36 \%$ (76/88) on AF ( Table-4).
Table 1: Age and sex distribution of tubercular lymphadenitis.

\begin{tabular}{|llll|}
\hline Age & Male & Female & Total \\
\hline $0-10$ & 2 & 3 & 5 \\
\hline $11-20$ & 9 & 17 & 27 \\
\hline $21-30$ & 15 & 18 & 33 \\
\hline $31-40$ & 3 & 8 & 11 \\
\hline $41-50$ & 3 & 4 & 7 \\
\hline $51-60$ & 2 & 2 & 4 \\
\hline $61-70$ & 1 & 1 & 2 \\
\hline Total & $35(39.77 \%)$ & $53(60.23 \%)$ & 88 \\
\hline
\end{tabular}

Table 2: Site distribution of tubercular lymph node.

\begin{tabular}{|ll|}
\hline Site & Number (\%) \\
\hline Cervical lymph node & $57(64.77 \%)$ \\
\hline Axillary lymph node & $8(9.09 \%)$ \\
\hline Supraclavicular lymph node & $4(4.55 \%)$ \\
\hline Cheek & $1(1.14 \%)$ \\
\hline Submandibular & $7(7.95 \%)$ \\
\hline Submental & $2(2.27 \%)$ \\
\hline Chest & $4(4.55 \%)$ \\
\hline Inguinal lymph node & $1(1.14 \%)$ \\
\hline Other & $4(4.55 \%)$ \\
\hline Total & 88 \\
\hline
\end{tabular}

Table 3: Cytomorphological patterns.

\begin{tabular}{|lll|}
\hline Cytomorphological patterns & Number & Percentage \\
\hline Granuloma & 75 & $85.23 \%$ \\
\hline Necrosis & 70 & $79.55 \%$ \\
\hline Lymphoid background & 21 & $23.86 \%$ \\
\hline Acute inflammation & 25 & $28.41 \%$ \\
\hline Giant cell & 15 & $17.05 \%$ \\
\hline Non documented & 4 & $4.55 \%$ \\
\hline
\end{tabular}

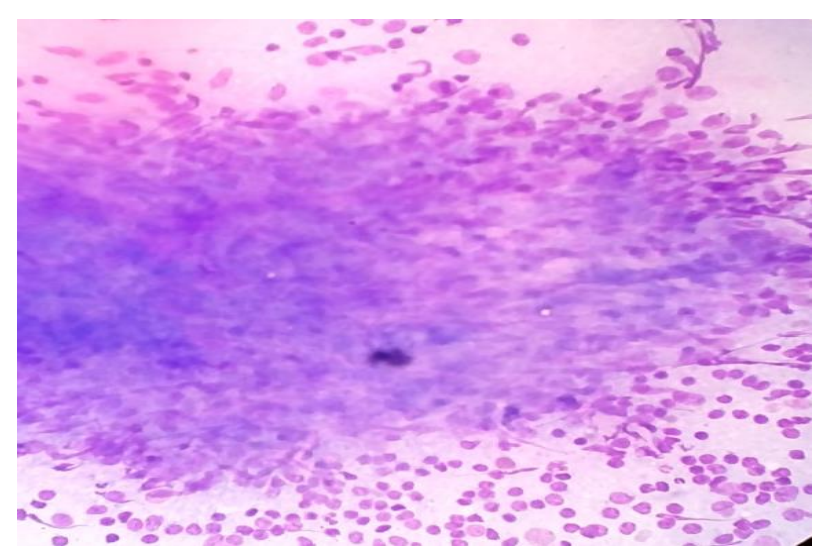

Figure 1: Well-formed granuloma in Giemsa stain 40x. 


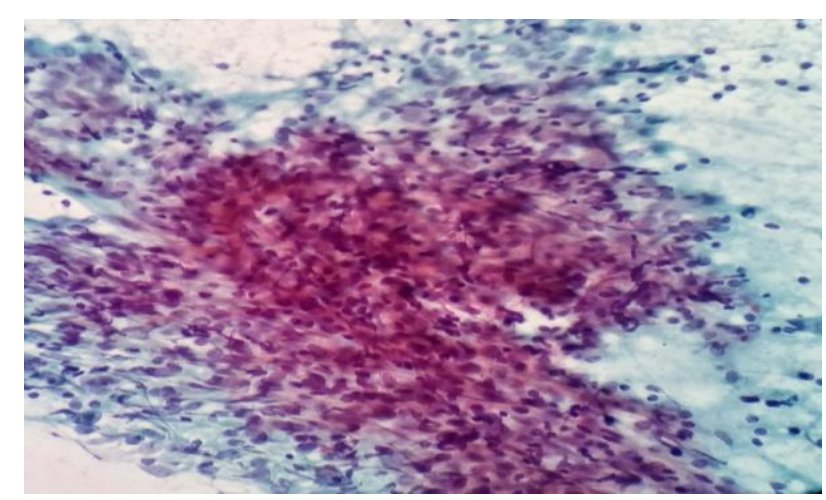

Figure 2: Well-formed granuloma in PAP stain 40x.

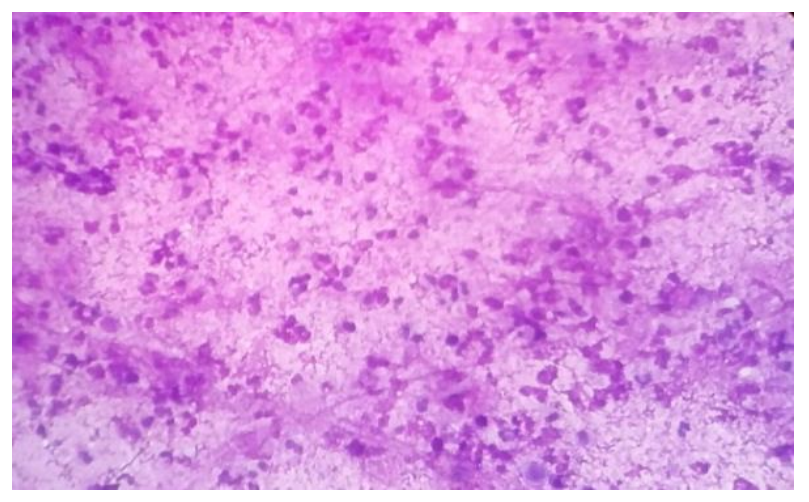

Figure 3: Acute inflammation with necrosis in Giemsa stain 40x.

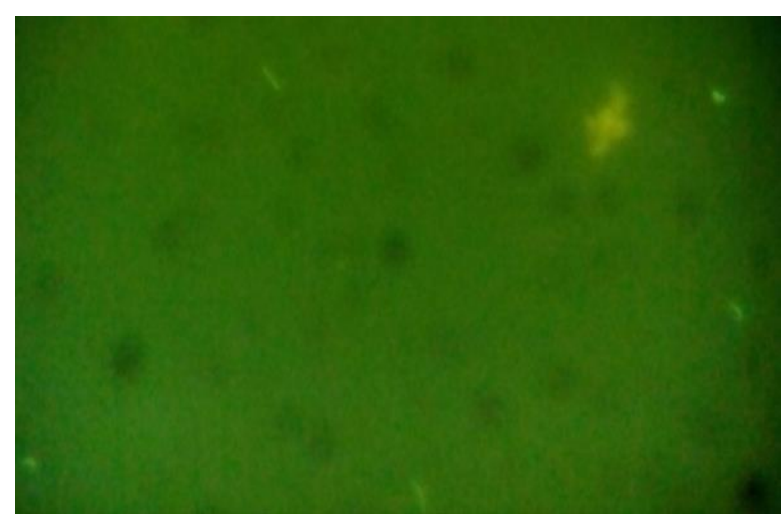

Figure 4: Demonstration of bacilli on auraminerhodamine stained smear under fluorescent microscopy using blue excitation filter 40x.

Table 4: Comparesion of detection of AFB by ZiehlNeelsen stain, auramine-rhodamine stain and autofluorescence.

\begin{tabular}{|lcll|}
\hline $\begin{array}{l}\text { Cytomorphology } \\
\text { tubercular } \\
\text { lymphadenitis }\end{array}$ & $\mathrm{Zn+}$ & $\mathbf{A R +}$ & $\mathrm{AF}+$ \\
\hline 88 & $33(37.5 \%)$ & $72(81.82 \%)$ & $76(86.36 \%)$ \\
\hline
\end{tabular}

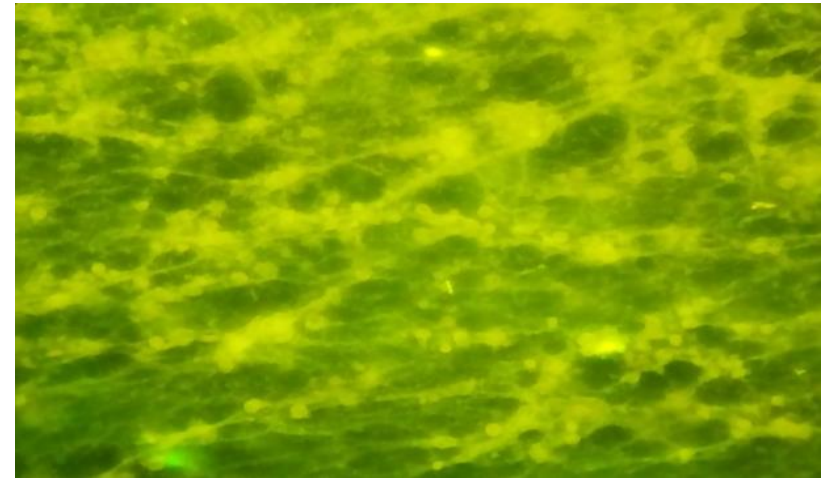

Figure 5: Demonstration of bacilli through autofluorescence technique on papanicolaou stained smears (PAP) 40x.

\section{DISCUSSION}

In many low and middle income countries with limited resources, the diagnosis of $\mathrm{TB}$ is still based on poorly validated symptom-based algorithms, often not resulting in a definitive diagnosis. The clinical presentation of tuberculosis is usually fever, night sweat, weight loss, anorexia. But some time delay in diagnosis has often been attributed to atypical clinical presentation and radiological presentation. The diagnosis of tuberculosis by cytomorphology is not new. It is a necrotizing granulomatous infection, which cytologically demonstrates the microscopic equivalent of caseous necrosis, granular-appearing necrotic background, together with mature lymphocytes, epithelioid histiocytes and multinucleated langhans type histiocytes. Several conditions, including mycosis, bacterial and viral adenitis can present the same cytology as does mycobacterium tubercular adenitis does. Laboratory tests may be essential to establish the cause of such adenopathy correctly, because treatment and prognosis may differ. Demonstration of Mycobacterium tuberculosis in fine needle aspirates becomes necessary for an early and accurate treatment. Fine needle aspiration cytology provides a rapid and definitive tissue diagnosis in the superficial lymphadenopathy. This study demonstrates that it also permits confirmation of the presence of mycobacteria with AFB stain in microscopy. The diagnosis of tuberculosis is confirmed by the demonstration of tubecular bacilli. Mycobacteria are slender rod shaped, non-motile, non-sporing, aerobic bacterium measuring 2 to $10 \mathrm{um}$ in length. It has lipid coat which makes it difficult to stain but once stained cannot be decolourised with alcohol. Thus, termed as acid fast bacilli (AFB) as they retain carbon fuschin staining (AFB stain or $\mathrm{ZN}$ stain) even after washing with acid alcohol. Flourscence staining utilize same approach as $\mathrm{ZN}$ staining but carbon fuschin is replaced by flourscent dye as auramine which as primary stain followed by counter stain (potassium permanganate) employed to highlight stain organism for easier recognition for the diagnosis of tubercular bacilli in the samples examined. A comparison between $\mathrm{ZN}, \mathrm{AR}$ and $\mathrm{AF}$ was done in the 
present study. It was found that autofluorescence was more sensitive for identifying the bacilli. There were only two cases which showed ARS positivity on smear but were negative by AF. In comparison to both, AF on PAP stained smears provides a safe, inexpensive and exposure free diagnostic procedure along with more positive results. Even this technique does not require any addition to the standard PAP stain. In other studies on FNA smears of lymph nodes, autofluorescence was found to be more sensitive than AR and ZN staining. ${ }^{9-11}$

Autofluorescence is simple, sensitive and inexpensive, it is not widely used. It requires a fluorescent microscope, which may not be readily available, but has the advantage of not requiring additional stains and is therefore inexpensive and rapid. ${ }^{8,9}$ If the cytomorphology is consistent with mycobacterial infection, and the organism is identified by $\mathrm{ZN}$ staining, AR or AF, the probability of a false positive diagnosis is small, and patients may safely commence therapy. FNAC of superficial lymph nodes is an outpatient procedure and requires little infrastructure and equipment and is therefore ideal for resource limited countries. The basic diagnostic modalities of cytomorphology and subsequent morphological identification of the organism are readily available and relatively inexpensive.

\section{CONCLUSION}

AF staining is more sensitive than the auraminerhodamine fluorescent and $\mathrm{ZN}$ staining in demonstration of mycobacterium bacilli in fine needle aspiration cytology of tubercular lymphnode.

\section{ACKNOWLEDGMENTS}

Authors would like to thank the pulmonary medicine department.

Funding: No funding sources Conflict of interest: None declared

Ethical approval: The study was approved by the Institutional Ethics Committee

\section{REFERENCES}

1. Dandapat MC, Mishra BM, Dash SP, Kar PK. Peripheral lymph node tuberculosis: A review of 80 cases. Br J Surg. 1990;77(8):911-2.
2. Lau SK, Kwan S, Lee J, Wei WI. Source of tubercle bacilli in cervical lymph nodes: A prospective study. J Laryngol Otol. 1991;105(7):558-61.

3. David HL: bacteriology of the mycobacterioses. US department of health, education and welfare, PHS, CDC, Washington DC; 1976.

4. Daniel TM. Rapid diagnosis of tuberculosis: Laboratory techniques applicable in developing countries. Rev Infect Dis. 1989;2(Suppl 2):471-8.

5. Balows A, Hausler WJ, Hermann KL, Shadomy HJ. Manual of clinical Microbiology. $5^{\text {th }}$ ed. Washington: D.C: American society for Microbiology 1991:308-11.

6. Savic B, Sjobring U, Alugupalli S, Larsson L, Miorner H. Evaluation of polymerase chain reaction, tuberculostearic acid analysis, and direct microscopy for the detection of Mycobacterium tuberculosis in sputum. J Infect Dis. 1992;166(5):1177-80.

7. Jain A, Bhargava A, Agarwal SK. A comparative study of two commonly used staining techniques for acid fast bacilli in clinical specimens. Indian Journal of Tuberculosis. 2002;49:161-2.

8. Wright CA, van Zyl Y, Burgess SM, Blumberg L, Leiman G. Mycobacterial autofluorescence in papanicolau stained lymph node aspirates: a glimmer in the dark? Diagnostic Cytopathology. 2004;30(4):257-60.

9. Wright CA, M. Burg MV, Geiger D, Noordzij JG, Burgess SM,Marais BJ. Diagnosing mycobacterial lymphadenitis in children using fine needle aspiration biopsy: cytomorphology, ZN staining and autofluorescence -making more of less. Diagnostic Cytopathology. 2008;36(4):245-51.

10. Joshi P, Singh M, Bhargava A, Singh M, Mehrotra R. Autofluorescence- an important ancillary technique for the detection of Mycobacterium tuberculosis: revisited. Diagnostic Cytopathology. 2012;41(4):330-4.

11. Thakur B, Mehrotra R, Nigam JS. Correlation of various techniques in diagnosis of tuberculous lymphadenitis on fine needle aspiration cytology. Pathology Research International. 2013;2013: http://dx.doi.org/10.1155/2013/824620.

Cite this article as: Krishna M, Kumar A. Tuberculous mycobacteria bacilli fluorescence and compare with Ziehl- Neelsen stain in fine-needle aspiration cytology of tubercular lymphnode. Int J Otorhinolaryngol Head Neck Surg 2016;2:66-9. 